\title{
Composition, Distribution, and Characterization of Polycyclic Aromatic Hydrocarbons in Soil in Linfen, China
}

\author{
S. Fu $\cdot$ H. X. Cheng $\cdot$ Y. H. Liu $\cdot$ X. J. Xia \\ X. B. Xu
}

Received: 30 March 2008/Accepted: 22 August 2008/Published online: 5 September 2008

(C) Springer Science+Business Media, LLC 2008

\begin{abstract}
A total of 10 surface soil samples representing the entire area of Linfen City were collected and analyzed for the presence of 16 polycyclic aromatic hydrocarbons. The total polycyclic aromatic hydrocarbon concentration ranged from 1.1 to $63.7 \mu \mathrm{g} \mathrm{g}^{-1}$. Analysis of the sources of contamination revealed that polycyclic aromatic hydrocarbons in the soil were derived from combustion sources. Specifically, the primary source of polycyclic aromatic hydrocarbons was coal combustion, but the samples were also effected to varying degrees by traffic emissions. Furthermore, increased levels of contamination were observed in northeast Linfen due to the distribution of industrial plants.
\end{abstract}

Keywords Contamination - Linfen $\cdot$ Polycyclic aromatic hydrocarbons $\cdot$ Soil

Polycyclic aromatic hydrocarbons (PAHs) are an important class of organic pollutants that have received considerable attention due to their carcinogenic and mutagenic properties (Blackburn and Kellard 1986). As a result, sixteen

S. Fu · X. J. Xia $\cdot$ X. B. Xu (ه)

State Key Laboratory of Environmental Chemistry and Ecotoxicology, Research Center for Eco-Environmental

Sciences, Chinese Academy of Sciences, P.O. Box 2871,

18 Shuangqing Road, Haidian District,

Beijing 100085, China

e-mail: xuxb1006@sina.com

H. X. Cheng · Y. H. Liu

Institute of Geophysical and Geochemical Exploration, Chinese

Academy of Geological Science, Langfang 065000, China un-substituted PAHs are included in the US Environmental Protection Agency (EPA) list of priority pollutants that require monitoring. For these reasons, many studies have recently been conducted to evaluate PAHs (Durjava et al. 2007). However, relatively little data regarding PAHs in China is currently available (Ma et al. 2005), even though such data is essential to enable reliable calculations of the global stock of PAHs in the environment.

Linfen, which is located in the southwest Shanxi Province, China, occupies an area of $20,275 \mathrm{~km}^{2}$. The urban area in the central part of Linfen contains 339.8 thousand residents. The area is dominated by a temperate semi-wet monsoon climate, and the mean annual temperature is $12.2^{\circ} \mathrm{C}$. China clay is the primary representative soil type in Linfen. In addition, Linfen is the heavy chemical industry base of China; therefore, there are many industrial plants within a few kilometers of the urban area of Linfen. In recent years, Linfen has been producing a higher volume of chemical products, as well as secondary energy products such as coke and electric power. In addition, due to recent growth the energy consumption by all types of industrial activities in Linfen has increased.A large amount of waste gas or particles containing PAHs are often emitted into the atmosphere as a result of incomplete combustion during industrial activities. These PAHs are then transferred to soil via deposition (Vogt et al. 1987; Trapido 1999). Even though contamination has likely increased in Linfen due to the recent increase in industrial activities, $\mathrm{PAH}$ residues in the soil of Linfen have never been investigated in detail. Little data regarding levels of PAHs in the soils of Linfen is available; therefore, this study was conducted to evaluate the spatial distribution of PAHs in Linfen. In addition, we attempted to identify the sources of pollution, and explored possible factors affecting contamination to prevent further environmental deterioration in Linfen. 


\section{Materials and Methods}

Surface soil samples $(0-10 \mathrm{~cm})$ were obtained from 5 urban sites and 5 industrial plants in Linfen City, Shanxi Province (Fig. 1) in January 2006. The urban soil samples were composites comprised of 9 sub-samples in a corresponding grid on a map of the sampling sites. Each industrial plant soil sample was a composite comprised of 3 sub-samples. All samples were collected from the surface soil in triplicate using a hand-held coring device. Overlying vegetation was removed prior to collection of the sample. The gathered samples were then freeze-dried, thoroughly mixed, passed through a 60 mesh sieve, transferred to a pre-cleaned amber glass container and then maintained at $4^{\circ} \mathrm{C}$ until further analysis. The remaining water content in the soil was determined gravimetrically after drying the individual composite samples in an oven at $105^{\circ} \mathrm{C}$ for $12 \mathrm{~h}$. All results were reported on a dry weight basis.

A mixed PAH standard solution $\left(1,000 \mu \mathrm{g} \mathrm{mL}^{-1}\right)$ that included naphthalene (Na), acenaphthylene (Acy), acenaphthene (Ace), fluorene ( $\mathrm{Fl})$, phenanthrene (Phe), anthracene (An), fluoranthene (Flu), pyrene (Pyr), benz[a]anthracene (BaA), chrysene (Chr), benzo[b]fluoranthene $(\mathrm{BbF})$, benzo[k] fluoranthene $(\mathrm{BkF})$, benzo[a]pyrene $(\mathrm{BaP})$, indeno[1,2,3cd]pyrene (InP), dibenz[a,h]anthracene (DBA), and benzo [g,h,i]perylene (BghiP) was purchased from Sigma Aldrich, St. Louis, MO (Milwaukee, WI, USA). In addition, 2-flurobiphenyl (2-FBP), which was used as a surrogate standard, was purchased from Supelco (Bellefonte, PA, USA). The standards were then diluted to the desired concentration using isooctane and then used as working standards. Silica gel (100200 mesh) was purchased from Qingdao Haiyang Chemical Co. (Qindao, China) and then activated in a drying oven at $550^{\circ} \mathrm{C}$ for $6 \mathrm{~h}$. Anhydrous sodium sulfate (Beijing Chemical
Factory, China) was heated at $600^{\circ} \mathrm{C}$ for $12 \mathrm{~h}$ prior to being used to eliminate organic contamination. All solvents used were of pesticide grade and were purchased from J. T. Baker (Phillipsburg, NJ, USA).

Five grams of each sample were weighed accurately and then ground into a free-flowing powder with anhydrous sodium sulfate with a mortar and pestle. The samples were then extracted with $30 \mathrm{~mL}$ of hexane/acetone $(1: 1, \mathrm{v} / \mathrm{v})$ by ultrasound at $3,000 \mathrm{~g}$ for $4 \mathrm{~min}$, after which they were centrifuged and the supernatant removed. This process was repeated three times, after which 2 -FBP was added as a surrogate standard. The concentrated extracts were then evaporated to $1 \mathrm{~mL}$ under a gentle $\mathrm{N}_{2}$ stream prior to purification. The PAHs were then purified using a chromatography column $(30 \mathrm{~cm} \times 10 \mathrm{~mm}$ i.d. $)$ containing $6 \mathrm{~g}$ of activated silica gel and $2 \mathrm{~g}$ of anhydrous sodium sulfate. The fraction containing the PAHs was eluted using $25 \mathrm{~mL}$ hexane/dichloromethane $(3: 2, \mathrm{v} / \mathrm{v})$, after which the solvent was evaporated to $100 \mu \mathrm{L}$ under a gentle $\mathrm{N}_{2}$ stream and then stored for subsequent analysis.

The PAHs were analyzed using an Agilent 6890 gas chromatograph equipped with flame ionization detector (FID). Separation was performed on a $30 \mathrm{~m} \mathrm{DB}-5(30 \mathrm{~m} \times$ $0.25 \mathrm{~mm}$ i.d., $0.25 \mu \mathrm{m}$ film thickness) capillary column. The injector and detector temperatures were 280 and $300^{\circ} \mathrm{C}$, respectively. After the sample was injected, the $\mathrm{GC}$ column was maintained at $70^{\circ} \mathrm{C}$ for $2 \mathrm{~min}$, then increased by $4^{\circ} \mathrm{C}$ $\mathrm{min}^{-1}$ to $280^{\circ} \mathrm{C}$ and held at this temperature for $15 \mathrm{~min}$. The total run time was $54.5 \mathrm{~min}$. Quantification of the samples was then performed using an external standard method.

To confirm the PAH results, selected typical samples were evaluated using an Agilent 6890 series gas chromatograph coupled to an Agilent 5973 mass spectrometer (MS) using an electron impact ionization source (EI) in the
Fig. 1 Sampling sites of surface soil in urban areas of Linfen

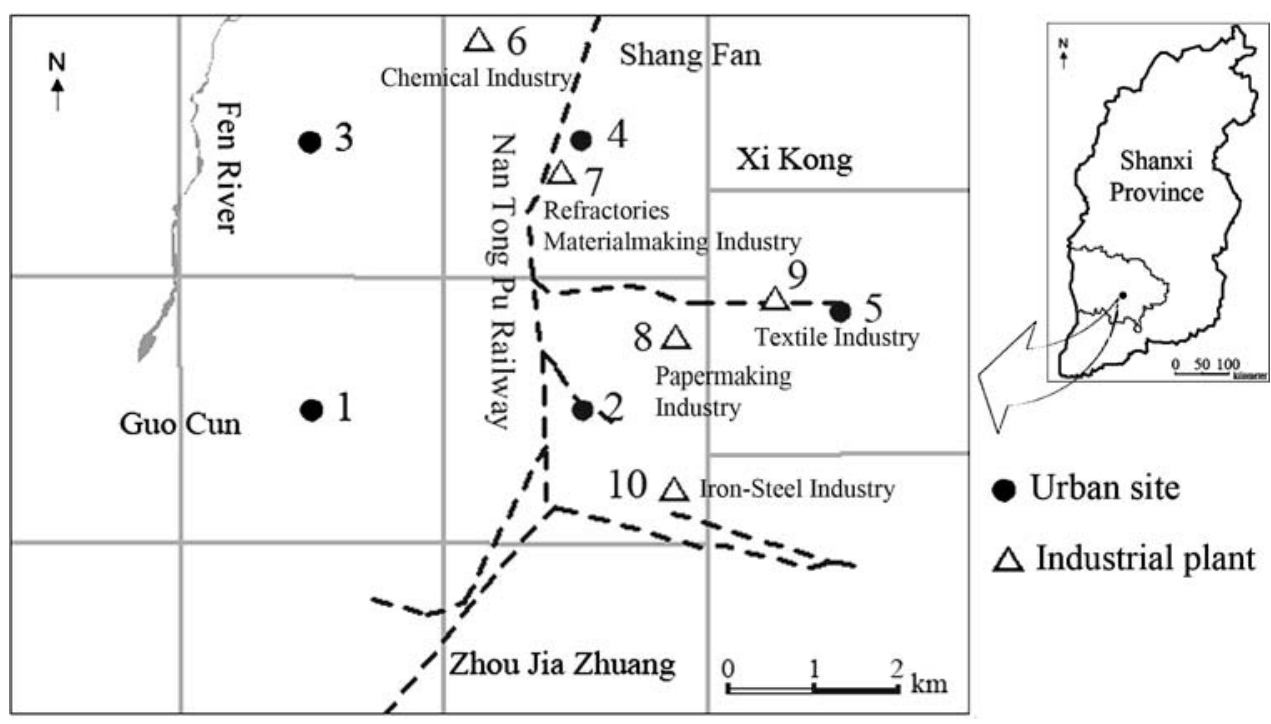


selected ion monitoring (SIM) mode. In EI mode, the MS source temperature was $230^{\circ} \mathrm{C}$, the transfer line was $300^{\circ} \mathrm{C}$ and the electron energy was $70 \mathrm{eV}$. Gas chromatographic separation was then conducted under the conditions described above.

In addition, a laboratory method control group was analyzed to demonstrate that the samples were not affected by interference or cross-contamination. Furthermore, a procedural blank was run in parallel with every set of six samples to check for interference and cross-contamination. Moreover, duplicate samples were analyzed in the laboratory as an additional quality control tool to ensure the validity of the results. The instrument stability and relative response factor variance were also measured by analyzing the calibration standard solutions during each sample batch.

Identification of PAHs was confirmed, after which the concentrations were measured using an external quantification standard consisting of known amounts of each of the target compounds. For accuracy and precision of analysis, method blanks were run first using the same solvents that were used when the actual samples were run. No contaminant PAHs were found in the method blanks $(n=3)$. The average recovery was determined in triplicate by spiking blanks with known concentrations of the standards. The limits of detection (LOD) were calculated as three times the response of the signal-to-noise $(\mathrm{S} / \mathrm{N})$ ratio and the limits of quantification (LOQs) were calculated as five times the $\mathrm{S} / \mathrm{N}$ ratio. These parameters are shown in Table 1. Additionally, each of the soil samples was spiked with a known concentration of 2-FBP as a surrogate standard prior to extraction to compensate for the loss of components. The recoveries of the 2-FBP surrogate ranged from $70 \%$ to $95 \%$. The recoveries of the surrogate were satisfactory and no correlation of analytical data was applied to the sample results.

Table 1 Limits of detection (LODs), limits of quantification (LOQs), recoveries and relative standard deviations (RSD\%) of soil samples analyzed using the method described herein $(n=3)$

\begin{tabular}{|c|c|c|c|c|c|c|c|c|}
\hline & $\mathrm{Na}$ & Acy & Ace & $\mathrm{Fl}$ & $\mathrm{Ph}$ & An & Flu & Pyr \\
\hline LODs (ng $\mathrm{g}^{-1}$ ) & 2.1 & 1.8 & 1.8 & 2.3 & 2.2 & 1.9 & 2.2 & 2.1 \\
\hline LOQs (ng g $\left.{ }^{-1}\right)$ & 3.6 & 3.0 & 3.0 & 3.8 & 3.7 & 3.2 & 3.7 & 3.4 \\
\hline Recoveries (\%) & 92 & 91 & 90 & 98 & 103 & 95 & 100 & 101 \\
\hline \multirow[t]{2}{*}{ RSD $\%$} & \pm 7 & \pm 3 & \pm 6 & \pm 9 & \pm 2 & \pm 3 & \pm 9 & \pm 6 \\
\hline & $\mathrm{BaA}$ & Chr & $\mathrm{BbF}$ & $\mathrm{BkF}$ & $\mathrm{BaP}$ & InP & DBA & Bghip \\
\hline LODs (ng g $\left.{ }^{-1}\right)$ & 2.5 & 1.5 & 2.1 & 1.8 & 2.0 & 2.0 & 2.1 & 1.9 \\
\hline LOQs (ng g ${ }^{-1}$ ) & 4.2 & 2.5 & 3.5 & 3.0 & 3.3 & 3.4 & 3.6 & 3.2 \\
\hline Recoveries (\%) & 105 & 76 & 105 & 90 & 100 & 101 & 104 & 97 \\
\hline RSD $\%$ & \pm 4 & \pm 7 & \pm 3 & \pm 7 & \pm 3 & \pm 4 & \pm 7 & \pm 5 \\
\hline
\end{tabular}

\section{Results and Discussion}

All 16 PAHs were identified in each of the urban and industrial plant soil samples, which indicates wide occurrence of these compounds in Linfen city. The total PAH concentration (defined as the sum of the 16 PAHs) ranged from 1.1 to $2.8 \mu \mathrm{g} \mathrm{g}^{-1}$ (median: $1.9 \mu \mathrm{g} \mathrm{g}^{-1}$, dry weight) in urban soil and 16.8-63.7 $\mu \mathrm{g} \mathrm{g}^{-1}$ (median: $31.8 \mu \mathrm{g} \mathrm{g}^{-1}$, dry weight) in industrial plant soil (Fig. 2). The median concentrations in the soil from the industrial plant were much higher than the concentrations from the soil in urban areas.

When compared with other areas in China, the concentration of the $\Sigma$ PAHs in the soil from Linfen was higher than those in surface soil in Beijing (0.017-0.132 $\left.\mu \mathrm{g} \mathrm{g}^{-1}\right)$ (Ma et al. 2005). Furthermore, the median concentration of PAHs in Linfen $\left(9.8 \mu \mathrm{g} \mathrm{g}^{-1}\right)$ was much higher than that of soil from the exurbs of Beijing (0.058-0.14 $\left.\mu \mathrm{g} \mathrm{g}^{-1}\right)$ (Ge et al. 2004), urban and rural soils in Tianjin $\left(457 \pm 1.46 \mathrm{ng} \mathrm{g}^{-1}\right)$ (Duan et al. 2005), vegetable soil in Guangzhou Province (160-370 ng g ${ }^{-1}$ ) (Cai et al. 2007), suburban soil in Dalian City (407 $\pm 78 \mathrm{ng} \mathrm{g}^{-1}$ ) (Wang et al. 2007) and surface soil in Hong Kong (7-410 $\mu \mathrm{g} \mathrm{g}^{-1}$ ) (Wang et al. 2006). In total, the results of this study revealed that soils in Linfen were contaminated to different degrees by polycyclic aromatic hydrocarbons.

As shown in Fig. 2, the concentration of the $\Sigma$ PAHs in the soil samples are higher in northeast Linfen. This may be due to the distribution of industrial plants, which are primarily located in the northeast portion of the city. The industrial activities in Linfen result in the consumption of a large amount of energy. This energy consumption results in waste gas or particles that contain PAHs produced as a result of incomplete combustion being emitted into the atmosphere. These PAHs are subsequently transferred to the soil via deposition (Vogt et al. 1987). Taken together, these factors could explain the increased tendency of contamination in the northeast areas of Linfen.

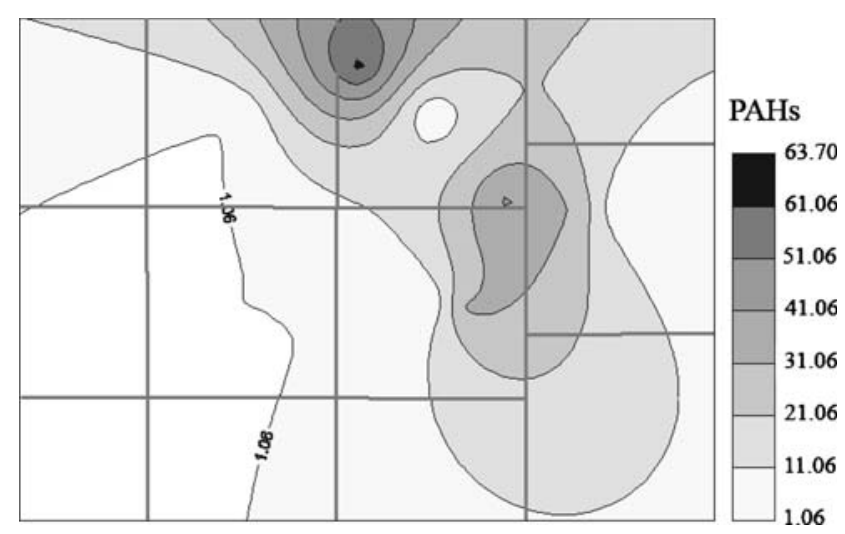

Fig. 2 The distributions of PAHs in surface soil in urban areas of Linfen (data in the figure are in $\mu \mathrm{g} \mathrm{g}^{-1}$ ) 
The composition of PAHs produced by different sources varies significantly. Therefore, the characteristic spectrum of PAHs can be used to identify their sources. The ratios of the sum of the major combustion specific compounds ( $\Sigma \mathrm{COMB}$, including Flu, Pyr, BaA, Chr, BbF, BkF, BaP, InP and Bghip) to the sum of the 16 EPA-PAHS ( $\Sigma$ COMB/ $\Sigma$ EPA-PAHs $)$, as well as the $\mathrm{An} /(\mathrm{An}+\mathrm{Phe})$ ratio and the $\mathrm{Flu} /(\mathrm{Flu}+\mathrm{Pyr})$ ratio are generally used to identify the sources of PAHs (Table 1) (Zhang et al. 2004). In general, combustion induces the production of relatively higher concentrations of $\Sigma \mathrm{COMB}$; therefore, a large proportion of $\Sigma C O M B$ is characteristic of PAHs that originated from combustion processes (Zhang et al. 2004). Additionally, most crude oil samples produce a Flu/(Flu + Pyr) ratio of $<0.40$, while coal combustion generates a Flu/(Flu $+\mathrm{Pyr}$ ) ratio of $>0.40$ (Zhang et al. 2004). In the present study, the $\Sigma \mathrm{COMB} / \Sigma$ EPA-PAH ratios varied from 0.66 to 0.81 , which indicates that the PAHs in the soil originated from combustion activity. Furthermore, the $\mathrm{Flu} /(\mathrm{Flu}+\mathrm{Pyr})$ ratio ranged from 0.54 to 0.63 , which further suggests that coal combustion was the primary source of PAHs in the soil in Linfen.

The $\mathrm{BaP} / \mathrm{BghiP}$ ratio can be used to distinguish PAHs produced by traffic exhaust from those produced by coal combustion. A ratio of $\mathrm{BaP} / \mathrm{BghiP}$ between 0.3 and 0.44 indicates that the PAHs originate from traffic exhausts, while a ratio of 0.9-6.6 indicates that the PAHs originate from coal combustion (Sawicki 1962). Furthermore, a Flu/ (Flu + Pyr) ratio of $<0.40$ is characteristic of the combustion of liquid fossil fuels (traffic emission), whereas a ratio of $>0.4$ indicates that the PAHs originate from grass, wood or coal combustion (Zhang et al. 2004). In the present study, the ratio of $\mathrm{BaP} / \mathrm{BghiP}$ ranged from 0.60 to 4.00 , with $40 \%$ of the values falling between 0.9 and 6.6. In addition, $100 \%$ of the samples had a Flu/(Flu + Pyr) ratio of $>0.4$. Furthermore, a plot of $\mathrm{BaP} / \mathrm{BghiP}$ versus Flu/ $($ Flu + Pyr) revealed that $10 \%$ of the samples fell in the combustion zone (I), which suggests that the majority of PAHs in the soils originated from the combustion of coal (Fig. 3). Additionally, $90 \%$ of the samples had a Flu/ (Flu + Pyr) ratio of $>0.4$ and a $\mathrm{BaP} / \mathrm{BghiP}$ ratio of $<0.9$, which is characteristic of a mixture of coal combustion and traffic emission. Taken together, these results indicate that the PAHs in the soil originated from combustion, with the primary source being coal combustion, but that traffic emissions also influenced the presence of PAHs.

This study evaluated the compositions and distribution of PAHs in soils collected from urban areas in Linfen. The results of this study indicate that the contamination status of the PAHs is greater in northeast Linfen, possibly as a result of the distribution of industrial plants. Furthermore, analysis of the sources of contamination revealed that PAHs in the soil were derived from a combustion source.

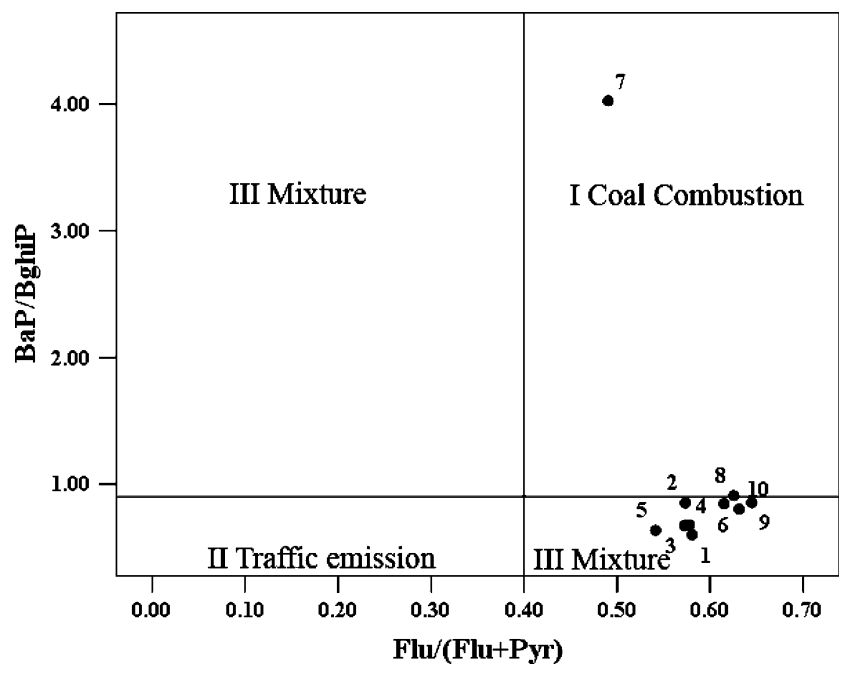

Fig. 3 PAH cross plot for the ratios of Flu/(Flu + Pyr) versus BaP/ BghiP in surface soil in urban areas of Linfen

Specifically, these PAHs were primarily produced by coal combustion, although traffic emission also influenced the concentrations of PAHs in Linfen. Overall, there were various concentrations of PAHs in the soils in Linfen. Furthermore, these findings indicate that the status of PAHs should be monitored continuously, and that greater attention should be paid to industrial plants and areas.

Acknowledgment This study was supported by the National Natural Scientific Foundation of China (No. 20707031).

\section{References}

Blackburn GM, Kellard B (1986) Chemical carcinogens-part II. Chem Ind 20:687-695

Cai QY, Mo CH, Li YH, Zeng QY, Katsoyiannis A, Wu QT, Ferard JF (2007) Occurrence and assessment of polycyclic aromatic hydrocarbons in soils from vegetable fields of the Pearl River Delta, South China. Chemosphere 68:159-168. doi:10.1016/j. chemosphere.2006.12.015

Duan YH, Tao S, Wang XJ, Li BG, Xu FL, Li WX, Cao J, Zhu LZ, Luo YM (2005) Spatial distribution and source of PAH in Tianjing's top soil. Acta Pedologica Sinica 42:492-497

Durjava MK, ter Laak TL, Hermens JLM, Struijs J (2007) Distribution of PAHs and PCBs to dissolved organic matter: high distribution coefficients with consequences for environmental fate modeling. Chemosphere 67:990-997. doi:10.1016/j.chemo sphere.2006.10.059

Ge XL, Xie WM, Luo SG (2004) Distribution and composition of polycyclic aromatic hydrocarbons in soil of Miyun and Fangshan area, Beijing. J Rock Miner Anal 23:132-136

Ma LL, Chu SG, Wang XT, Xu XB (2005) Polycyclic aromatic hydrocarbons in the surface soil from outskirts of Beijing, China. Chemosphere 58:1355-1363. doi:10.1016/j.chemosphere.2004. 09.083

Sawicki E (1962) Analysis of airborne particulate hydrons - their relative proportion affected by differents types of pollution. J Polycyel Aromat Comp 22:23-25 
Trapido M (1999) Polycyclic aromatic hydrocarbons in Estonian soil: contamination and frofiles. Environ Pollut 105:67-74. doi: 10.1016/S0269-7491(98)00207-3

Vogt NB, Brakstad F, Thrane K (1987) Polycyclic aromatic hydrocarbons in soils and air: statistical analysis and classification by the SIMCA method. Environ Sci Technol 21:35-44. doi: 10.1021/es00155a003

Wang XJ, Liu RM, Wang KY, Hu JD, Ye YB, Zhang SC, Xu FL, Tao S (2006) Application of multivariate spatial analysis in scalebased distribution and source study of PAHs in the topsoil: an example from Tianjin, China. Environ Geol 49:1208-1216. doi: 10.1007/s00254-005-0165-1

Wang Z, Chen J, Qiao X, Yang P, Tian F, Huang L (2007) Distribution and sources of polycyclic aromatic hydrocarbons from urban to rural soils: a case study in Dalian, China. Chemosphere 68: 965-971. doi:10.1016/j.chemosphere.2007.01.017

Zhang ZL, Huang J, Yu G, Hong HS (2004) Occurrence of PAHs, PCBs and organochlorine pesticides in Tonghui River of Beijing, China. Environ Pollut 130:249-261. doi:10.1016/j.envpol.2003. 12.002 